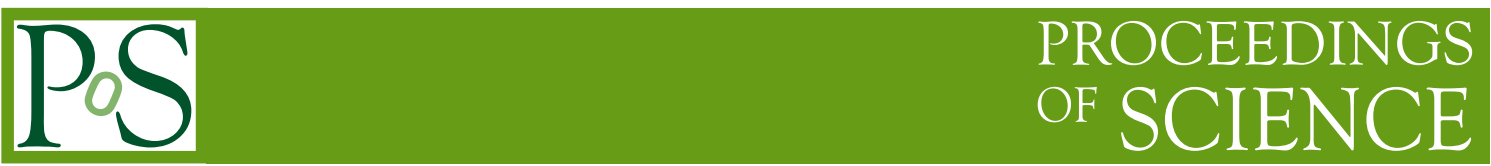

\title{
Consequences of Early Thermalization at Low and High $p_{T}$
}

\section{Peter Steinberg*}

Brookhaven National Laboratory

Upton, NY 11973, USA

E-mail: peter.steinbergabnl.gov

\begin{abstract}
The systematics of bulk entropy production in experimental data on $\mathrm{A}+\mathrm{A}, p+p$ and $e^{+} e^{-}$interactions over a wide range of energy and rapidity are presented. It is proposed that scenarios with very early thermalization, such as Landau's hydrodynamical model, capture several essential features of the experimental results. It is also pointed out that the dynamics of systems which reach the hydrodynamic regime give similar multiplicities and angular distributions as those calculated in weak-coupling approximations (e.g. pQCD) over a wide range of beam energies. Finally, predictions for the LHC based on the assumption of early, rapid thermalization are presented.
\end{abstract}

High-pT physics at LHC

March 23-27 2007

University of Jyväskylä, Jyväskylä, Finland

\footnotetext{
*Speaker.
} 


\section{Introduction}

When considering the complex multiparticle state produced in RHIC collisions, it is now commonplace to assume that the system thermalizes rapidly and is described by the equations of hydrodynamics [1]. It is generally assumed that the longitudinal expansion is born into the "scaling" regime where $v_{z}=z / t$, as was suggested by Bjorken in 1983 [2]. This makes the hydrodynamic equations much simpler, reducing the numerical complexity of the solutions. It is also something which was expected in the parton model approach of Feynman and Bjorken. However, it is often forgotten that the hydrodynamic approach to strongly interacting system was invented almost thirty years before the Bjorken paper, and considered a much different initial state. The so-called "Landau Hydrodynamical Model" [4, 5, 19, 6, 9] has the same assumptions for the hydrodynamic evolution (most importantly, zero viscosity), but has very different assumptions for the initial velocity gradient.

The 2+1D hydro of Bjorken, adapted to modern contexts by a wide range of authors, starts with a picture of partial stopping of the projectiles with the produced matter reflecting the internal structure of the nucleons. If the partons in the nucleon followed a structure function $F(x) \propto 1 / x$, this quite naturally would provide a boost invariant initial condition if all of the partons were suddenly put on-shell and assumed to be in local equilibrium (since $d y=x d x$, so if $d N / d y=C$, then $d N / d x=C / x$ ). However, it has been experimentally established more than a decade ago by HERA that there is an enormous number of low $x$ gluons. Thus, boost-invariance is not an obvious feature of the initial state. The situation is not helped by a lack of knowledge about the intrinsic thermalization time, which is usually assumed to be $O(1 \mathrm{fm} / \mathrm{c})$ but has not yet been determined experimentally.

Landau's model [4] (identical to Fermi's [3] in the initial state) is the opposite extreme. The the matter is not born into any particular velocity distribution but is born at rest, of course with a huge energy density. While there are no obvious ways to achieve this in perturbative calculations, it does not violate energy/momentum conservation. In this picture the only scales entering the problem are the CMS energy $\sqrt{s}$ and the Lorentz-contracted volume of the projectiles $V / \gamma$. Given this, the only time-scale in the problem is $O(R / \sqrt{s})$, the Lorentz-contracted radial dimension of the projectiles. This scale determines the thermalization time, and thus the energy density, entropy, etc. More importantly, since boost-invariance is not assumed, then the dynamics is non-trivially $3+1 \mathrm{D}$. The most stark example of non-trivial behavior in 3+1D at RHIC comes from the data on the elliptic flow parameter $v_{2}$ as a function of $\eta$. There is a clear and strong rapidity dependence, not dissimilar to the inclusive charged particle production, suggestive of real longitudinal physics. Of course, non-ideal hydrodynamical effects have been invoked to explain this (e.g. Ref.[7], but the point of this work is to suggest that there may be more ways to approach this problem. The rest of this proceedings will be to explore the consequences of Landau's initial conditions on various aspects of RHIC (and possibly LHC) phenomena.

\section{Elementary Collisions}

Compared to RHIC collisions, "elementary" collisions of protons and antiprotons, or electrons and positrons, which produce many hadrons seem to look quite sparse. Thus, they are generally 

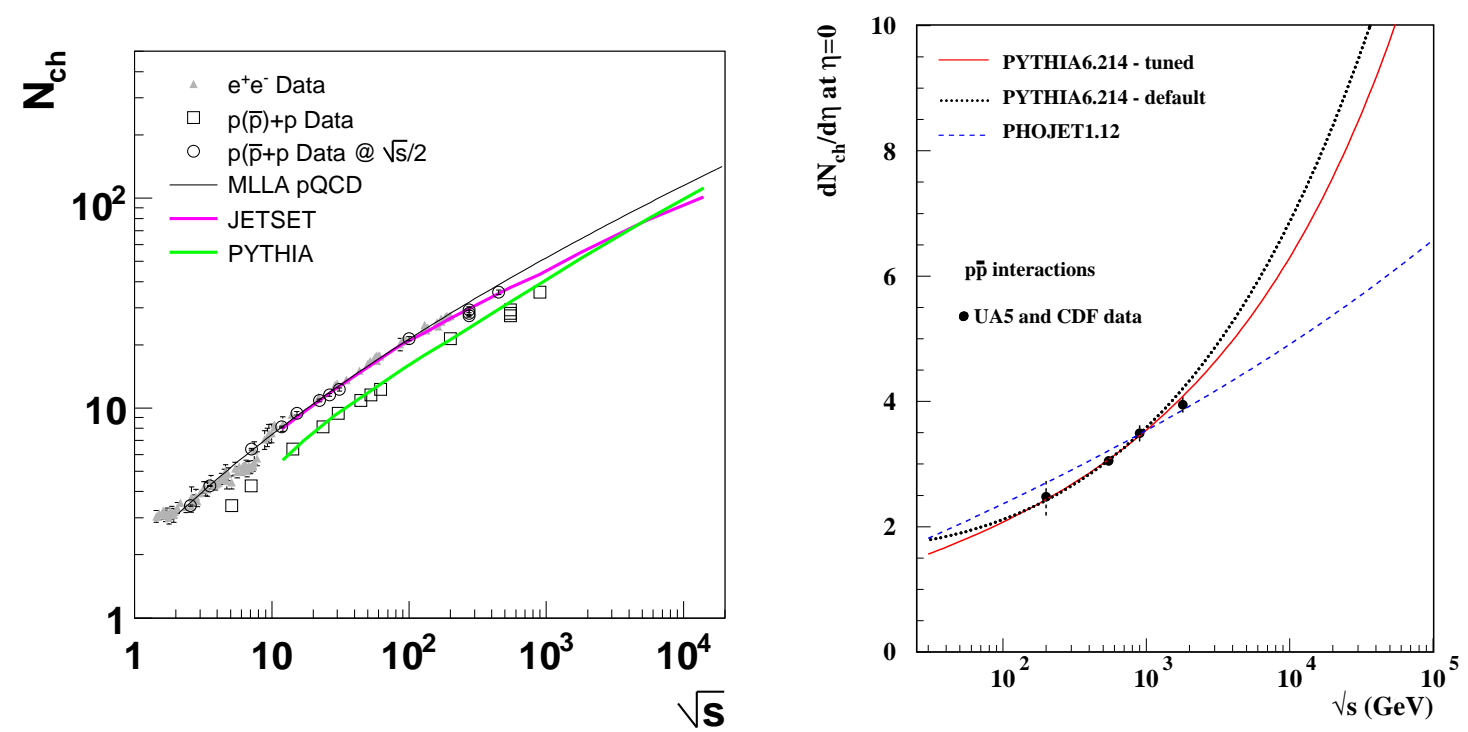

Figure 1: (left) Total primary charged particle multiplicity for $e^{+} e^{-}$(with up to $10 \%$ admixture of weak decays) and $p(\bar{p})+p$ collisions, compared with MLLA pQCD calculations, JETSET, and PYTHIA. (right) Midrapidity density for $\bar{p}+p$ collisions vs. $\sqrt{s}$ compared with PYTHIA and PHOJET, from Ref. [10]

thought to result from very different physics processes.

Electron-positron annihilation into hadrons used to be understood using concepts based on the original "string models" of the 1970's, with extensions incorporating multiple gluon production [8]. More recently, purely perturbative calculations involving gluon ladders can capture many features of the data, down to details of jet fragmentation. This is especially true of calculations of the total multiplicity, which have good descriptive and predictive power, starting from the early SPEAR data up to the top LEP2 energies. A full accounting of the running coupling in jet fragmentation gives formulae that scale as $n_{c h} \propto \alpha_{s}^{A} \exp (B \sqrt{\ln (s)})$ [乌]. One achieves similar results in various "parton cascade" approaches, such as JETSET, which augments the older string models with perturbative gluon emission.

Collisions of protons and antiprotons are generally understood in a "two-component" scenario. The soft component is thought to be the domain of "non perturbative QCD" but understood phenomenologically by means of descriptive features like longitudinal phase-space and limited $p_{T}$. Various implementations of this can be tuned to describe the available data. Of course, jet phenomena have been observed as the energies increased, suggesting that there is a separate "hard" component at work in $\mathrm{p}+\mathrm{p}$ collisions. This has been successfully modeled by combining the structure functions measured at high- $Q^{2}$ in $e+p$ collisions, with $\mathrm{pQCD}$ cross sections to get the angular distributions, and fragmentation functions measured in $e^{+} e^{-}$reactions used to parameterize the relationships between the outgoing quarks and gluons and the measured hadrons.

Various models incorporate the hard and soft components in different schemes, such as PYTHIA[11], HERWIG[12], PHOJET [13], and HIJING[14]. And yet, despite being based on similar inputs, most of these models predict different extrapolations of existing data to high energies [10], as 

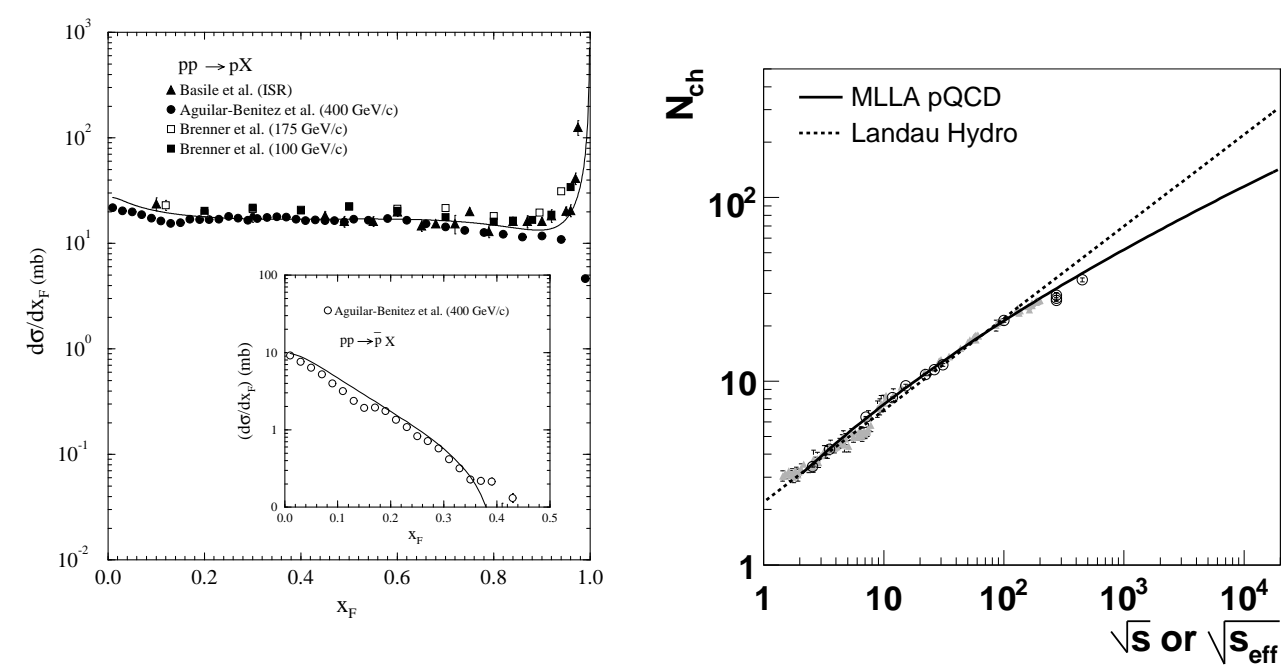

Figure 2: (left)Inclusive $d N / d x_{F}$ for protons $p+p$ collisions at several energies. Inset shows the same for anti-protons. From Ref. [18]. (right) $N_{c h}$ for $e^{+} e^{-}$at $\sqrt{s}$ (grey triangles) and $p(\bar{p})+p$ at $\sqrt{s_{\text {eff }}}=\sqrt{s} / 2$ (open circles) compared with MLLA pQCD and Landau Hydrodynamics. Both functions have been adjusted by an overall scale factor.

shown in Fig. 1. One expects an interesting early running of the LHC while the various models (or tunings thereof) are validated, or ruled out, by the first data.

One major uncertainty in understanding soft particle production in $\mathrm{p}+\mathrm{p}($ and $\bar{p}+p)$ collisions is related to the lack of dynamical mechanisms in the models. It is still not generally understood how the incoming baryons are "stopped", and their energy transmuted into particles[15]. The net rapidity loss of the incoming baryons has been studied extensively in fixed-target experiments as well as at the ISR (but not at the Tevatron collider, unfortunately) [16, 17]. It has been found that the distribution of $x_{F}=2 p_{Z} / \sqrt{s}$, the fraction of energy found in the outgoing "leading" particles is essentially flat (but with a quasi-elastic peak near $x_{F} \sim 1$ )[18], as shown in Fig. 2(left). More interestingly, this net baryon rapidity loss is found to correlate strongly with the total multiplicity, and approach the $e^{+} e^{-}$multiplicity measured at the same $\sqrt{s}[16]$. In fact, the $e^{+} e^{-}$and $p+p$ data overlap each other if $p+p$ is plotted at $\sqrt{s_{\text {eff }}}=\sqrt{s} / 2$. This suggests that 1 ) the net baryons measured in $\mathrm{p}+\mathrm{p}$ collisions reflect the inelasticity of the collision, and 2) the basic mechanisms of total entropy production in both $e^{+} e^{-}$and $p+p$ are quite similar.

One way to understand the similarity between the entropy produced in these two systems is by simply postulating that both are the result of an early, rapid equilibration process resembling the Landau scenario in its space-time profile. Cooper, Frye and collaborators [19] worked under this assumption in the 1970's. Thus, whatever complicated dynamics might be different between the two systems is rendered irrelevant by strong interactions between the fundamental constituent degrees of freedom. In that scenario, the rest of the evolution is isentropic and simply expresses the total entropy via the total multiplicity. Clearly, this is a difficult scenario to consider if one conceives of it proceeding via the kinetic equilibrium of the outgoing particles. However, it seems less problematic if the particles are thought to be the consequence of the freezeout of a fluid with many strongly-interacting degrees of freedom into the thousands of available mass states of QCD. 
This is a natural extension to the model that Fermi [ [ $]$ ] and Landau [ $[$ ] inadvertently proposed in the 1950's.

\section{Landau's Hydrodynamical Model}

\subsection{Multiplicity Formula}

Fermi and Landau both arrived at a simple formula for the total multiplicity in the early 1950's[3, Ð1. The derivation simply assumes complete thermalization of the total energy $E=\sqrt{s}$ in a Lorentz-contracted volume $V=V_{0} / \gamma=V_{0} /\left(\sqrt{s} / 2 m_{N}\right)$, leading to an initial energy density $\varepsilon=s / 2 m_{N} V_{0}$, which increases quadratically with $\sqrt{s}$. Assuming the blackbody equation of state $p=\varepsilon / 3$ and the first law of thermodynamics $d \varepsilon=T d \sigma$, leads to a scaling of the entropy density as $\sigma \propto s^{3 / 4}$. Multiplying the entropy density by the volume gives a total entropy $S=\sigma V \propto s^{3 / 4} / s^{1 / 2} \propto$ $s^{1 / 4}$. This is the famous Landau-Fermi multiplicity scaling formula, which suggests that total multiplicities will scale as the square root of the CMS energy, $N \propto \sqrt{E_{c m}}$. For a more generic equation of state (e.g. $\left.\varepsilon=c_{s}^{2} p\right), N \propto E_{c m}^{(1 / 2)\left(1-c_{s}^{2}\right) /\left(1+c_{s}^{2}\right)}$ 19.

While the pQCD formula mentioned above, shown in Fig. 2 (right), does a good job for the $e^{+} e^{-}$data, the Landau-Fermi formula does an equally good job describing the high-energy data when tuned on the lower energy data. It also naturally explains the constant ratio between the $p+p$ and $e^{+} e^{-}$data at the same $\sqrt{s}$, since $\left(s_{e f f} / s\right)^{1 / 4}=\sqrt{1 / 2} \sim 70 \%$. Of course, it remains an open question how higher-energy data will turn out, given that the two formulae differ significantly at much higher energies (pQCD giving $N_{c h} \sim 100$ and Landau giving $N_{c h} \sim 160$ at LHC energies) and the $\bar{p}+p$ data already seems to trend below even the $\mathrm{pQCD}$ prediction shown above.

Of course, the dynamical evolution does not end with the initial equilibrated system postulated by the Landau-Fermi model. Landau correctly recognized that if such a system achieves local equilibrium (i.e. with vanishingly-small mean free paths), it will behave hydrodynamically [ $\Theta$, 5]. The blackbody EOS implies a locally-traceless stress energy tensor, and thus scale-free (i.e. conformal) dynamics. Thus, the evolution of the system is determined only by the scales imposed at the beginning (the energy and volume) and at the end (the familiar freezeout condition such that evolution stops at $\left.T=T_{c h}\right)^{1}$

Landau's well-known initial conditions are quite simple: an enormous energy density with no longitudinal motion, packed into a volume contracted along the $\mathrm{z}$ axis by $1 / \sqrt{s}$. Following the evolution analytically from its initial 1+1D expansion to the late $3+1 \mathrm{D}$ expansion (using various approximations along the way), he found that the rapidity distribution of the fluid elements at freezeout is described by a Gaussian distribution with variance $\sigma_{y}^{2}=(1 / 2) \ln \left(s / 4 m_{N}^{2}\right) \equiv L$. Cooper, Frye and Schonberg completed the modern interpretation of hydrodynamics by suggesting that the fluid elements are not particles but hadronic "fireballs" which decay isotropically in their own rest frame[19]. Carruthers and Duong Van found that Landau's model was a better fit to data than the boost-invariant scenarios popular at the time [21].

It is worth taking a few moments to remark on what the Landau model is: It is a 3+1D model which assumes rapid local equilibration and has no free parameters. It has two scales, $\sqrt{s}$ and

\footnotetext{
${ }^{1}$ This is not dissimilar to QCD calculations, which take as input a hard scale $Q$ and a self-generated cutoff $\Lambda_{Q C D}$. In fact, there are many intriguing similarities between hydrodynamics and field theory calculations, as pointed out by Carruthers 6
} 

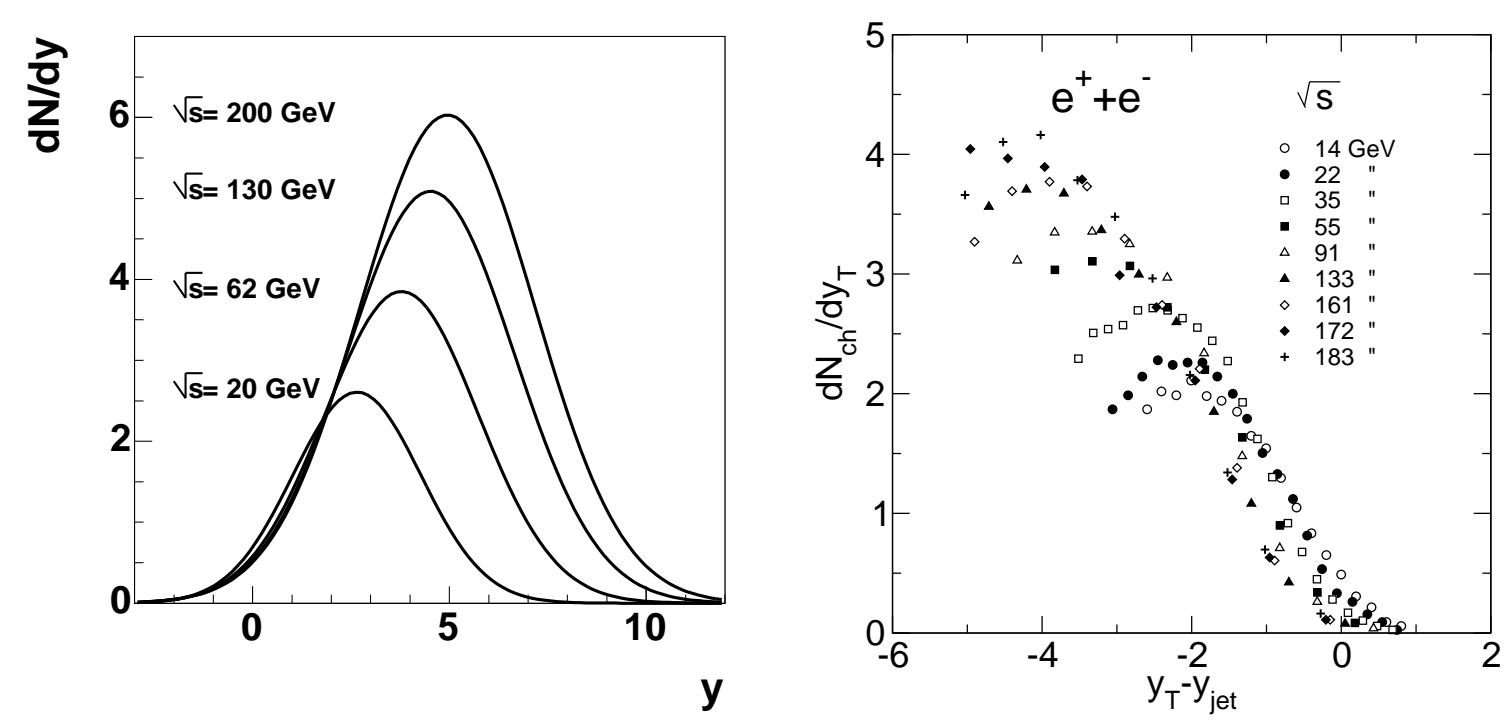

Figure 3: (left) Calculations of $d N / d y$ (with an arbitrary overall scale) the Landau hydrodynamic model (Equation 3.1), seen in the fixed target frame, showing "extended longitudinal scaling". (right) $d N / d y_{T}$, the rapidity distribution along the thrust axis in $e^{+} e^{-}$collisions, viewed in the frame of the outgoing quark $\left(y_{T}-y_{j e t}\right)$, from Ref.[23].

$T_{c h}$ which determine the initial and final states. Finally, it describes the energy dependence of the produced entropy and its angular distributions. There is no nuclear transparency in the model and no assumption of boost-invariance. Rather, the entire system is explicitly assumed to be in local thermal contact on asymptotically small time scales $t_{0} 1 / \sqrt{s}$ as the beam energy increases.

And a few words on what the Landau model is not: There is no description of net-baryon dynamics (or those of any conserved charges). There is no phase transition, but just a single EOS $p=\varepsilon / 3$. There is no hadronization per se, but just a simple freezeout criterion $\left(T=T_{c h}\right)$, and thus no mass dependence of $d N / d y$ (something which was discussed in the 1970's by Cooper and Frye) and certainly no resonance decays. Since these are clearly important pieces of physics, clearly seen in data, these issues should be seen as caveats for the various conclusions drawn later.

\subsection{Longitudinal Scaling}

One very non-trivial feature of Landau's hydrodynamical model appears when it is combined with the Landau-Fermi multiplicity formula

$$
\frac{d N}{d y}=K s^{1 / 4} \frac{1}{\sqrt{2 \pi L}} \exp \left(-\frac{y^{2}}{2 L}\right)
$$

and then viewed in the rest frame of one of the projectiles by making the transformation $y^{\prime}=$ $y-y_{\text {beam }}=y-(L+\ln (2))$, where $L=\ln \left(\sqrt{s} / 2 m_{P}\right)$

$$
\frac{d N}{d y^{\prime}} \sim \frac{1}{\sqrt{L}} \exp \left(-\frac{y^{\prime 2}}{2 L}-y^{\prime}\right)
$$



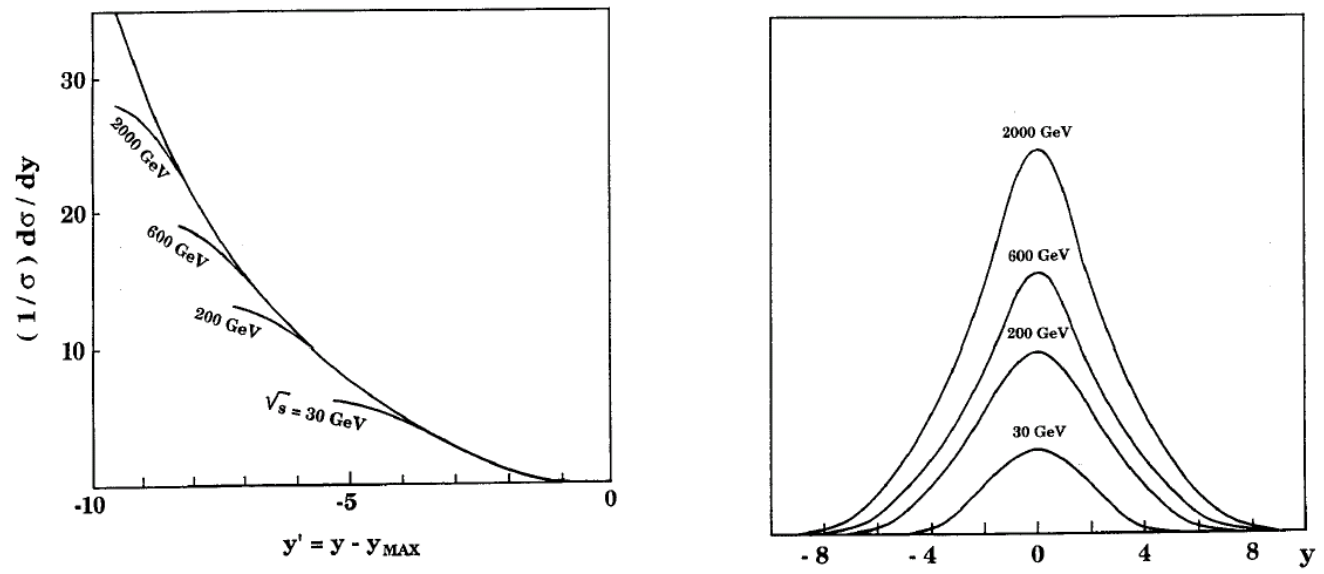

Figure 4: MLLA pQCD calculations, from Ref.[24], showing rapidity distributions in the rest frame of the outgoing quark (left) and in the CM frame (right).

One sees that as one approaches $y^{\prime}=0$ this becomes a function of $y^{\prime}$ alone, with some logarithmic scale breaking due to the $\sqrt{L}$. A direct plot of $d N / d y^{\prime}$ at several beam energies, shown in the left panel of Fig. 3, shows the phenomenon of "limiting fragmentation" or "extended longitudinal scaling" [20] even more clearly. While not an original observation about the Landau model (see e.g. Ref.[21]), this scaling was rediscovered in this context in Ref.[22]. This is a non trivial outcome of the formulae, and is even more intriguing considering that it is clearly seen in both $p+p$ and $\bar{p}+p$ data with respect to the beam axis, as well as $e^{+} e^{-}$data with respect to the thrust axis [23] as shown in the right panel of Fig. 3.

\subsubsection{Landau vs. pQCD}

But the surprises of Landau's model are not just limited to its relevance to experimental data. The calculations of jet fragmentation in perturbative QCD, in the MLLA framework discussed above, have been done by several authors during the 1980's. In Ref.[24], Tesima performed MLLA pQCD calculations (which have a different anomalous dimension than Mueller's, and thus presumably a different energy dependence) for the rapidity distribution of emitted gluons. He found that the rapidity was approximately Gaussian with a width scaling as $\sqrt{\ln (s)}$ and "translational invariance", seen by observing the fragmentation functions as a function of $y^{\prime}=y-y_{\max }$. Finally, we have already seen that the MLLA formula gives similar multiplicities to the Landau-Fermi formula over energy ranges for which data exist. Thus, we find that, even parametrically, pQCD and the Landau model can give similar results. Whether this is a particularly ornate accident, or whether the mathematics (non-Abelian gauge theories, and 3+1D hydrodynamics with Landau's initial conditions and freezeout criterion) share a deep underlying structure seems to be a particularly intriguing question.

The prevalence of extended longitudinal scaling in elementary collisions, and the predictions of this phenomenon from both MLLA pQCD and Landau's hydrodynamical model should not 


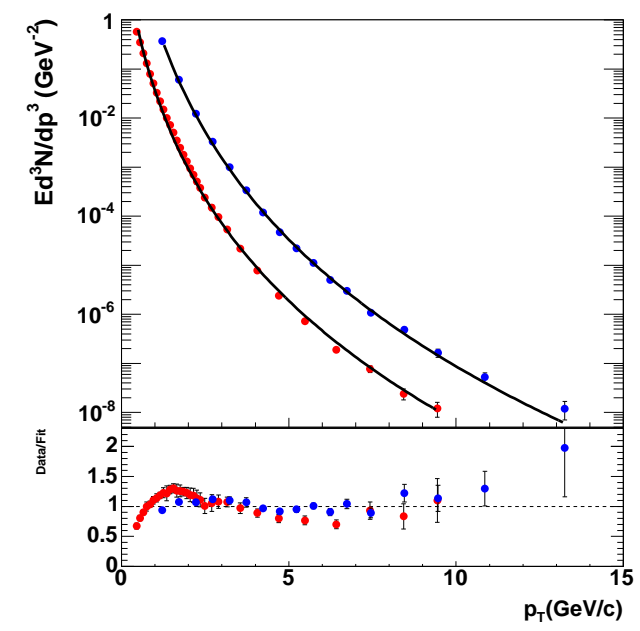

Figure 5: RHIC data on inclusive charged particles (STAR) and identified $\pi^{0}$ 's (PHENIX) at high $p_{T}$, compared with a fit to a Gaussian distribution in transverse rapidity.

be forgotten when discussing the phenomenon in $\mathrm{A}+\mathrm{A}$ in the context of newer theoretical frameworks, some of which will be discussed in the next section. The theoretical predictions for this scaling should also be kept in mind when trying to predict the shape of $d N / d \eta$ and the value of $d N / d \eta(\eta=0)$, e.g. in Ref.[25]. While the existing data suggest a "linear" trend to the limiting curve, the models shown here (both pQCD and Landau) suggest a nonlinearity as the energy increases, as seen in Figs 3 and 4 . This will be discussed in Section 5 .

\subsection{Transverse Distributions}

A final unexpected coincidence is seen in the transverse direction near $\mathrm{y}=0$. Carruthers and Duong-van noticed that the $p_{T}$ distribution of $\pi^{0}$ 's in $p+p$ collisions was well described out to $p_{T}=10 \mathrm{GeV}$ by a Gaussian distribution in transverse rapidity $y_{T}=\frac{1}{2} \ln \left(\frac{m_{T}+p_{T}}{m_{T}-p_{T}}\right)$ with $L \sim 0.51$ [26]. While no derivation was given for this phenomenological description, which holds over 10 orders of magnitude, an argument was made on a similar basis as for the Gaussian in the longitudinal direction. To see if this function continues to work well at RHIC energies, fits were made in Ref. [22] to PHENIX $\pi^{0}$ data [27] and STAR inclusive charged data [28] from $p+p$ collisions, which are shown in Fig. 5. Reasonable agreement is found with the STAR data with $L=0.56$, despite the combination of various particle species, and excellent agreement is found with the PHENIX data with $L=0.54$, up to $p_{T}=11 \mathrm{GeV}$. Above this value, the data starts to deviate from the Gaussian, which may imply that the agreement over 7 decades was an accident, or perhaps that different physics may become more important at very high $p_{T}$ (e.g. high- $x q q$ scattering vs. $g g$ ).

\section{Heavy Ion Collisions}

Moving from elementary collisions to heavy ion collisions brings in a large number of new dynamical considerations. The initial state should be characterized by shadowed parton distribution 


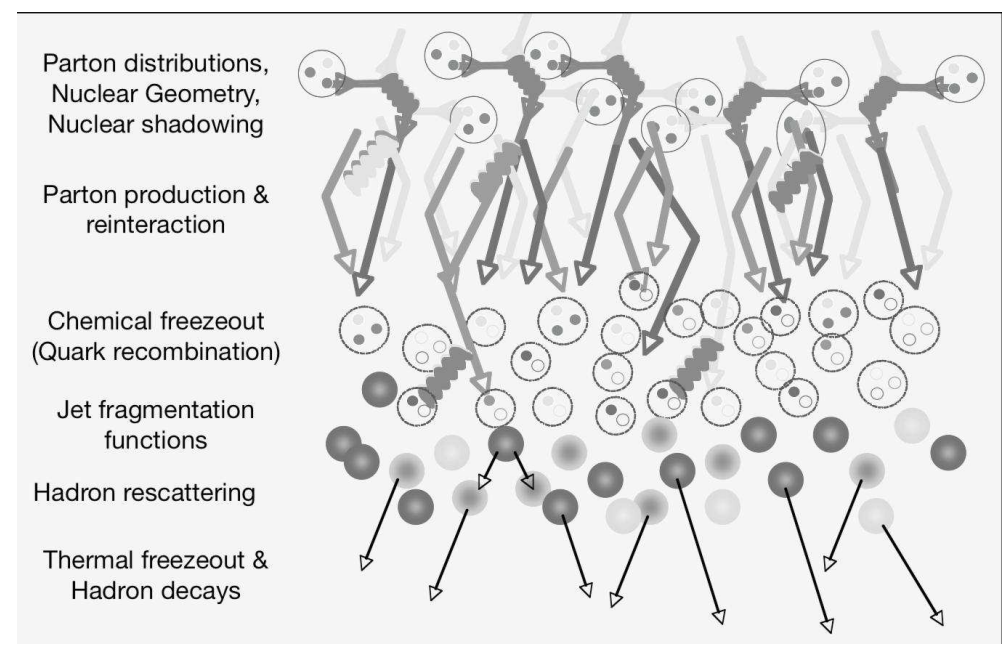

Figure 6: Schematic of various physics processes thought to be relevant over the time history of a heavy ion collision.

functions, as well as the nuclear geometry suggested by Glauber calculations. The early dynamics are driven by hard parton scattering and subsequent reinteractions, possibly leading to equilibration. Eventually the momentum transfers become low enough that hadron formation is preferred, and the quark chemistry freezes out, incorporating the thermalized quarks as well as the ones from jet fragmentation. These hadrons themselves may rescatter if the densities are sufficiently high, leading to an eventual thermal freeze-out. Finally, the final-state hadrons themselves decay, either immediately via strong processes, or over macroscopic distances via weak processes. All of these stages are in principle independent of the others, and thus could lead to a non-trivial energy and geometrical dependence as the relative contributions of soft and hard processes change (e.g. HIJING [14]) as well as rescattering in the partonic and/or hadronic phases [29].

And yet, it is not inconceivable that early thermalization may dominate the bulk observables like entropy production and angular distributions. Elliptic flow results at midrapidity already suggest high temperatures $\left(T_{0}>350 \mathrm{MeV}\right)$ at a thermalization time $\left(\tau_{0}<0.6 \mathrm{fm}\right)$ far smaller than hadronic time scales. There is no clear experimental signature measured to date that excludes the enormous energy density needed by the initial Landau/Fermi picture $\left(\sim 3 \mathrm{TeV} / \mathrm{fm}^{3}\right.$ at RHIC energies), except possibly thermal photon rates, which are beginning to be measured at RHIC. In fact, it has been noted that the rapidity distributions of pions measured by BRAHMS [30] and experiments at lower energies appear to be Gaussian (with possibly some flattening in $|y|<1$ ), as seen in Fig. 7, and approximately follow the Landau predictions from 1955.

The contributions of soft and hard processes are thought to be controlled by the number of participants and collisions respectively, which are typically calculated in a "Glauber" picture treating the nuclei as nucleons following classical paths. This scenario is based on QCD factorization theorems which imply that hard processes of partons become less sensitive to the soft components of the nucleon wave function as the momentum transfer grows (a consequence of asymptotic freedom). In the infinite momentum transfer limit, the probability of a hard processes should scale with the number of nucleons in the nuclear target seen by a projectile nucleon. Thus, the parameter 


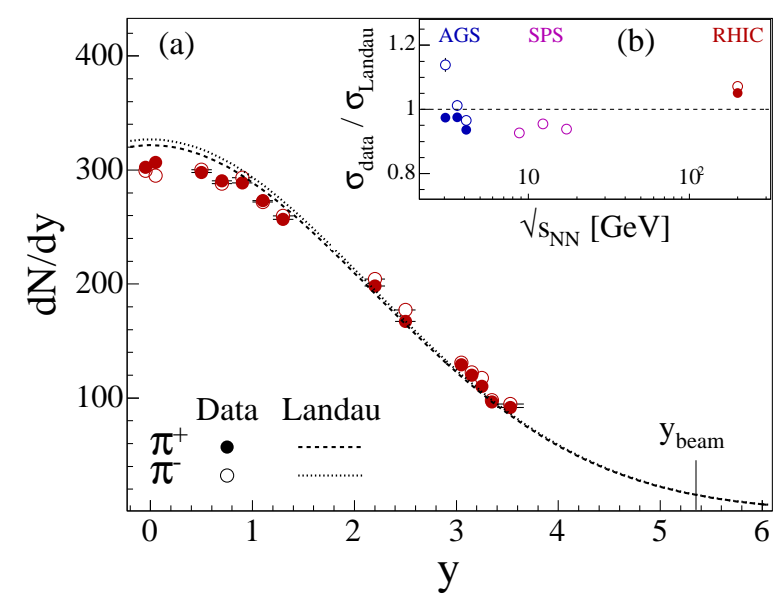

Figure 7: Rapidity distribution of pions measured in A+A by BRAHMS. The inset shows the comparisons of Gaussian fits over a wide range of $\sqrt{s_{N N}}$ divided by the Landau prediction $\sigma_{\text {Landau }}=\log \left(\sqrt{s} / 2 m_{P}\right)$.

$v \equiv N_{\text {coll }} /\left(N_{\text {part }} / 2\right)$ should be visible in the rates of high $p_{T}$ particles.

The inclusive ( $p_{T}$-integrated) yields of charged particles can be characterized by four "rules of thumb" (first laid out in Ref. [31]). These are useful concepts for describing what has been seen, but in general they should be used with care to extrapolate to higher energies, as they are not based on physical concepts.

- "log-rise" at $\eta=0$ : As seen in the left panel of Fig. 8, the inclusive particle density at mid-rapidity rises approximately logarithmically from AGS to top RHIC energies. This is different than the trend seen in $p+p$ collisions, which is more of a power-law, as shown in the same figure.

- limiting fragmentation: the right panel of Fig. 8 shows $d N / d \eta$ at four RHIC energies in a Lorentz frame with one of the projectiles at rest. One sees that the yields are invariant with energy when sitting at a fixed distance from beam rapidity, i.e. in $\eta^{\prime}=\eta-y_{\text {beam }}$.

- $N_{\text {part }}$ scaling: The limiting fragmentation phenomenon seems to hold true for all centralities, as shown in the right panel of Fig.9 Moreover, decreases at mid-rapidity appear to be correlated with increases at forward rapidities. These seem to average out to a near-constant value of $N_{c h} /\left(N_{\text {part }} / 2\right)$ as a function of $N_{\text {part }}$. Similar phenomena have been seen in $p+A$ collisions. This suggests a quite limited sensitivity to $v$, which is surprising in a "two-component" picture.

- Energy/Geometry Factorization: If limiting fragmentation holds true generally, and the peel-off from the universal curve is roughly at the same point, then the mid-rapidity density will depend only on centrality at all energies. This is seen in the right panel of Fig.9, where the relative changes at mid-rapidity as a function of centrality are found to be energyindependent. 

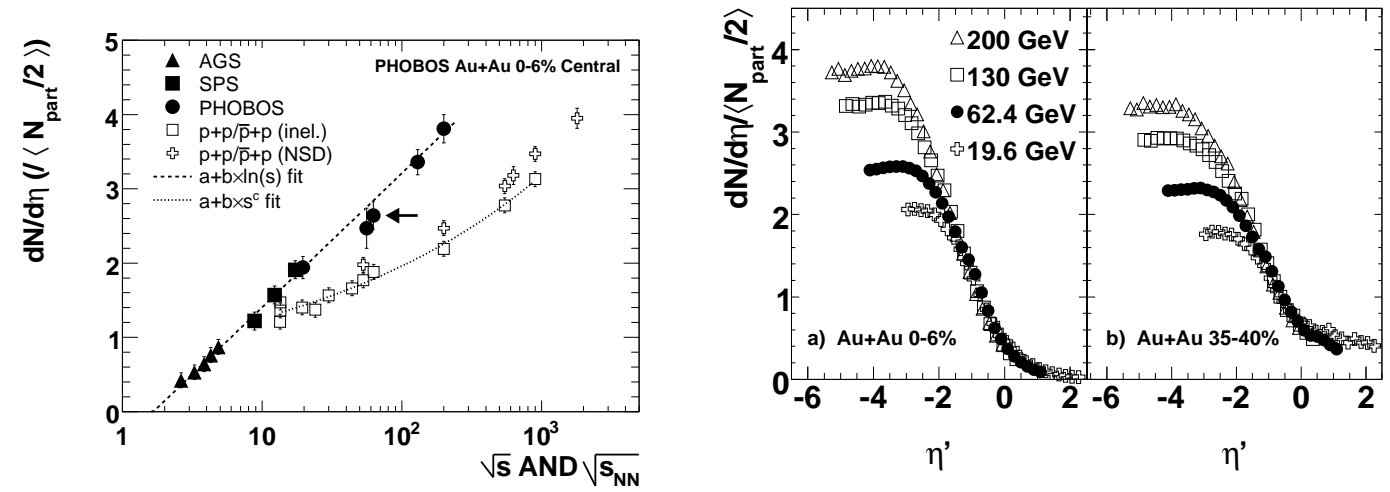

Figure 8: (left) Mid-rapidity multiplicity density, showing log rise (right) Extended longitudinal scaling for $\mathrm{Au}+\mathrm{Au}$ collisions at RHIC, from Ref.[31].
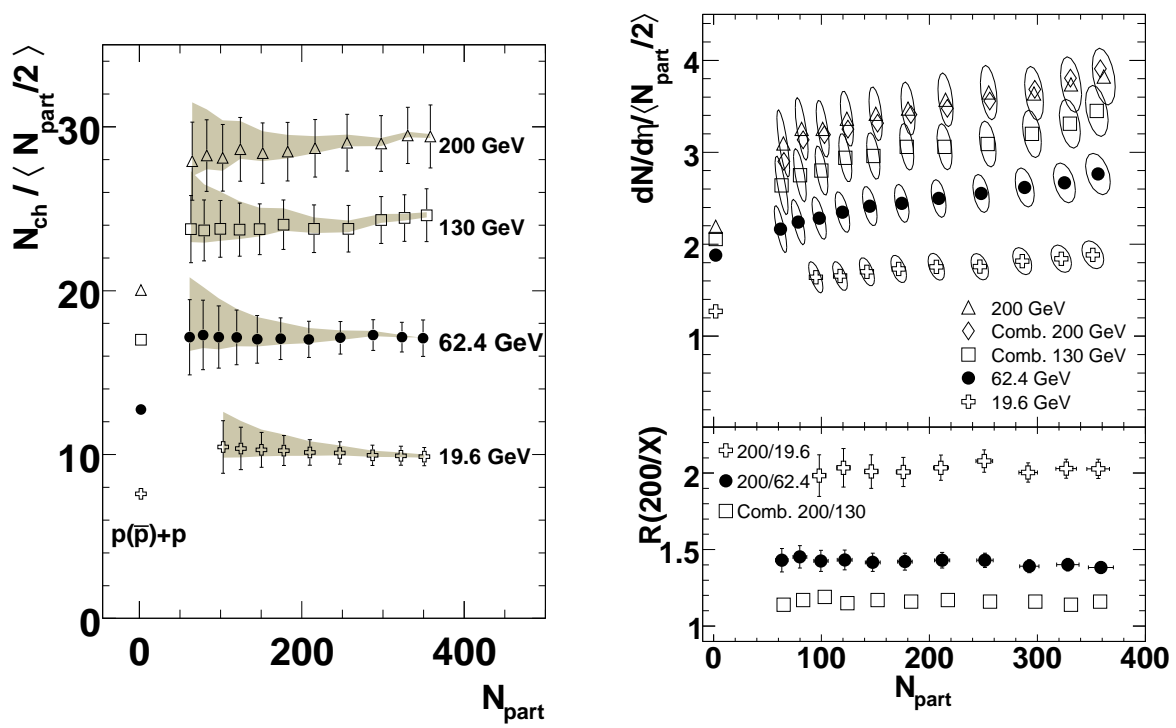

Figure 9: (left) Total multiplicity divided by the estimated number of participant pairs, as a function of $N_{\text {part }}$, from Ref.[31]. (right) Factorization of energy and centrality

These four rules of thumb are merely descriptive, empirical features of the multiplicity data. However, it is straightforward to make connections between them and Landau's hydrodynamical model. $N_{\text {part }}$ scaling is simply the scaling of the total entropy with the initial volume $V$ which scales linearly with the overlap. Limiting fragmentation is an "accidental", but non-trivial, consequence of the Fermi-Landau multiplicity formula and Landau's Gaussian rapidity distributions. The "logrise" is not inconsistent with the expected $s^{1 / 4} / \sqrt{\ln (s)}$ dependence, when one takes into account the role of the suppression of total entropy from the large baryon density at low energy, as proposed in Refs.[35], 36]. Finally, the factorization of energy and centrality might be understood if one treats the particle production in transverse cells as scaling with $N_{\text {part }}$ but shifted into the center of mass 

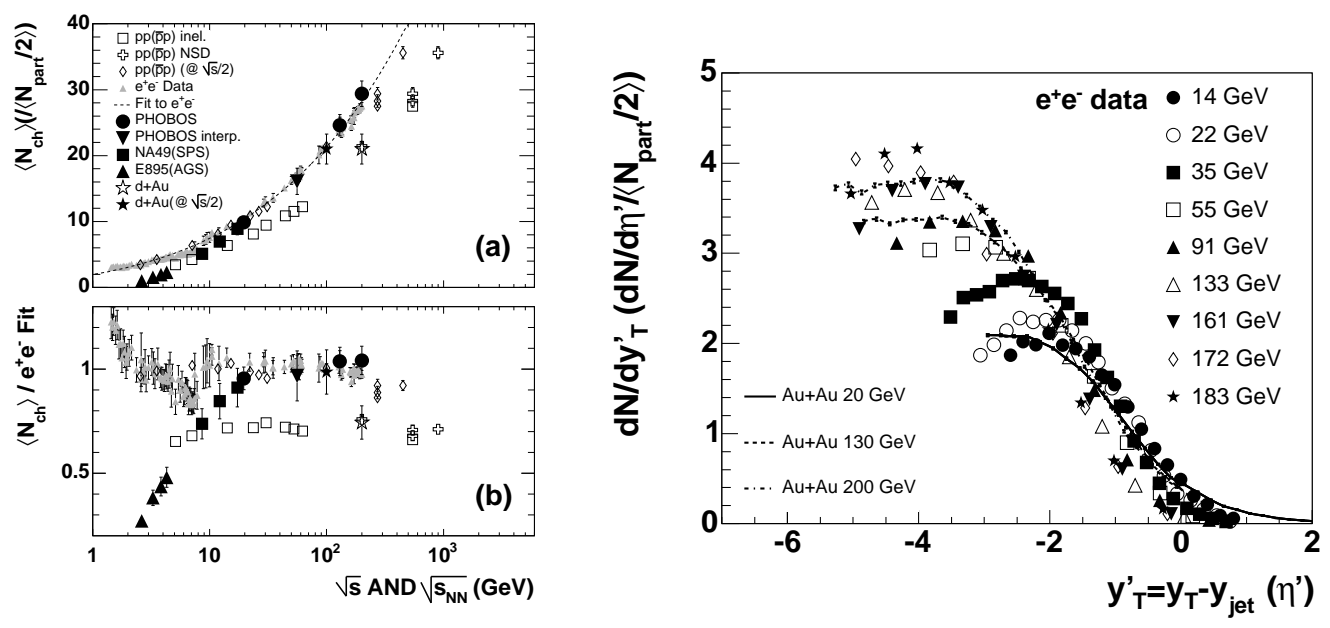

Figure 10: (left) Compilation of the total multiplicity for various systems, from Ref.[33], as well as the data divided by the MLLA-based fit to the $e^{+} e^{-}$data. (right) Heavy ion and $e^{+} e^{-}$data compared in the rest frame of one of the projectiles or jets (from Ref.[37]).

of the colliding tubes of nucleons, as was done for $\mathrm{d}+\mathrm{Au}$ collisions (as proposed in Ref. [32]).

The possible relevance of early thermalization in both $\mathrm{A}+\mathrm{A}$ and more elementary collisions makes it interesting to directly compare their entropies, as shown in the left panel of Fig. 10. The absence of a strong centrality dependence makes it possible to compare $N_{c h} /\left(N_{\text {part }} / 2\right)$ in A+A with other systems [33]. As discussed above, the $p+p$ data is similar to the $e^{+} e^{-}$if one takes the $\sqrt{s}$ to be an effective $\sqrt{s_{\text {eff }}}=\sqrt{s} / 2$, accounting for the average $x_{F}$ of the leading particles. This is assuming that the flat $d N / d x_{F}$ distribution is mainly comprised of baryons that do not participate in the thermalization or subsequent dynamical evolution. Conversely, it is found that $\mathrm{A}+\mathrm{A}$ and $e^{+} e^{-}$data are similar to one another between $\sqrt{s}=20-200 \mathrm{GeV}$ without any other adjustments except dividing by $N_{\text {part }} / 2$ [33], as shown in Fig.10. Given the previous comparisons of $p+p$ and $e^{+} e^{-}$, one particular efficient way to understand the comparisons with $\mathrm{A}+\mathrm{A}$ is to postulate that the multiple collisions experienced by each participant (as $v>2-3$ for all centralities considered in Ref. [33]) essentially stops all of the incoming energy. This alleviates the leading particle effect, and thus one finds the multiplicity per participant pair to be "universal" without additional scaling. Of course, to say that A+A data as showing "full stopping" requires some reinterpretation of the netbaryon rapidity distributions published by BRAHMS [34. This author has suggested elsewhere 36] that net-baryon rapidity distributions displaced from $y=0$ do not require partial stopping. Rather, they may be indicating how the stopped baryons are distributed in the thermalized matter in the longitudinal direction at the moment the hydrodynamic evolution begins.

These comparisons are somewhat mysterious if one considers $e^{+} e^{-}$reactions as involving just the physics of perturbative gluon radiation, while $\mathrm{A}+\mathrm{A}$ is usually discussed in terms of a stronglyinteracting partonic medium [38]. These two appear at first glance to be completely opposite limits of QCD physics, the very hard and very soft. However, it was mentioned above that parametrically, MLLA pQCD and Landau hydrodynamics are quantitatively very similar in their output, even if they do not appear to have similar functional forms. The Fermi-Landau scenario would also 


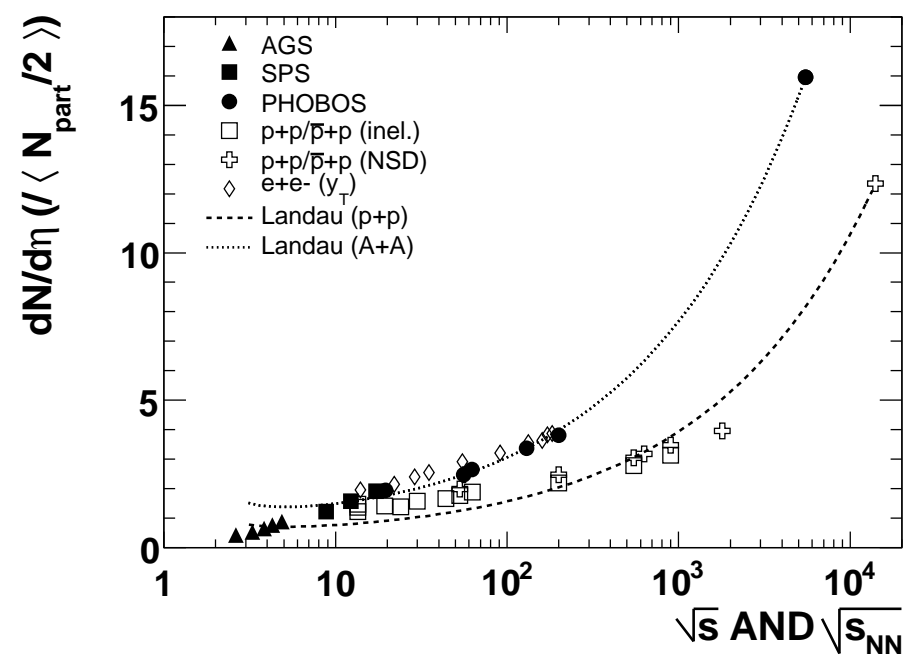

Figure 11: Midrapidity density for $\mathrm{A}+\mathrm{A}, p+p$ and $e^{+} e^{-}$as a function of $\sqrt{s}$ and $\sqrt{s_{N N}}$. Calculations of the Landau predictions are superimposed, each fit to high energy $\mathrm{A}+\mathrm{A}$ and $p+p$ data, with points showing the LHC predictions made in this work. The $\mathrm{A}+\mathrm{A}$ calculations agree reasonably while the $p+p$ fit is less satisfactory over the full range.

naturally predict that the multiplicity should scale linearly with the initial volume, which is clearly compatible with (and essentially predicted) the linear scaling of the total multiplicity with $N_{\text {part }}$ shown above. The same angular distributions as a function of $\sqrt{s}$ predicted by Landau also seem to appear in the elementary collisions as well, as does the phenomenon of limiting fragmentation (shown in the right panel of Fig. 10) which is a highly non-trivial outcome of Landau hydro and the Fermi-Landau statistical picture. Perhaps it is not necessary to use heavy nuclei to achieve local equilibration. It should be kept in mind that only the first radiations in a hard process are at a truly hard scale. Subsequent gluon emissions require the summations of successively more-complicated many-gluon diagrams, which perhaps drive the final distributions toward something resembling local equilibrium.

\section{Some Predictions for the $\mathrm{LHC}$}

The assumption of early, rapid thermalization can be used to make predictions for the LHC, as was done in Ref. [36]. From the basic formula, the midrapidity density scales as $\rho_{0}(\sqrt{s}) \propto$ $s^{1 / 4} / \sqrt{\ln \left(\sqrt{s} / 2 m_{P}\right)}$ - with no free parameters. Thus, the ratio of $\sqrt{s}=14 \mathrm{TeV}$ to $\sqrt{s}=200 \mathrm{GeV}$ in proton-proton collisions will be $\rho_{0}(14 \mathrm{TeV}) / \rho_{0}(200 \mathrm{GeV}) \sim 6.1$. The ratio of $\sqrt{s_{N N}}=5.5 \mathrm{TeV}$ to $\sqrt{s_{N N}}=200 \mathrm{GeV}$ for $\mathrm{A}+\mathrm{A}$ (where $\rho_{0}$ is scaled by $N_{\text {part }} / 2$ ) will be $\rho_{0}(5.5 \mathrm{TeV}) / \rho_{0}(200 \mathrm{GeV}) \sim 4.0$. This is shown in Fig.11, which includes $\rho_{0}$ for several types of collisions. Fits of the Landau energy dependence to data of each type (RHIC data for A+A, NSD UA5 data for $\bar{p}+p$ ) have been made, to account for the different $p_{T}$ distributions as well as the overall multiplicity scale.

It is interesting that while the formula gets the higher energy RHIC data, the description of the $p+p$ and $\bar{p}+p$ is less satisfactory, even qualitatively. Unfortunately, there may be several factors 
which could lead to this. Considering yields at mid-rapidity makes comparisons more sensitive to the details of particle production, both species and $p_{T}$ dependence. There are also issues to do with triggering, especially the contribution from diffractive events, which are not well understood theoretically, and are difficult to control experimentally unless one is actively measuring leading particles. These factors would certainly complicate a trivial application of the Landau formula for $d N / d y$ in a limited region of $d N / d \eta$. Clearly, the LHC will be an interesting place to test these ideas over a large range of $\sqrt{s}$.

\section{Summary and Outlook}

It is argued here that the concept of early thermalization has wide-ranging relevance in the study of multiparticle production. While it seems natural in the context of heavy ion collisions to believe that the system drives itself towards local equilibrium, it has often seemed difficult to accept the same conclusion for elementary collisions. However, the similar entropy density and longitudinal behavior seen in the full variety of systems makes it tempting to say that both A+A and $e^{+} e^{-}$evolve hydrodynamically. It then begs the question of what fundamental scale in QCD would prevent this statement from being true. This is intimately tied to the question of viscosity [39], as the latter concept is simply connected to the intrinsic mean free path of constituents [40]. Of course, all of this begs the question of how any of this reconnects with $\mathrm{pQCD}$, which seems to generate similar results for many observables sensitive to the dynamical evolution of the system (entropy, angular distributions, etc.).

Whatever the theoretical situation, detailed measurements of similar observables at higher energies or at high $\mu_{B}$ should provide crucial new information. The LHC will provide $p+p$ and A+A simultaneously, and FAIR at GSI will be specifically devoted to systems with large net baryon stopping and thus high $\mu_{B}$. In the high $\sqrt{s}$ sector, one will be testing the abilities of the system to thermalize, or not, on astoundingly short timescales of $O\left(10^{-} 3 \mathrm{fm} / \mathrm{c}\right)$. If the KSS bound is proven to be true in nature, this implies that the intrinsic length scale of the system can be no shorter than the thermal wavelength $\lambda$, which may run afoul of Landau's assumptions when $\lambda \propto 1 / \sqrt{m} s^{1 / 4}$ is greater than the Lorentz-contracted longitudinal dimension $\Delta z \propto\left(A^{1 / 3} 2 m_{p} / m_{\pi}\right) / s^{1 / 2}$. This may well happen at the LHC, depending quantitively on the thermal constituent mass $m$, rendering early thermalization impossible. In the high $\mu_{B}$ sector one may be able to explore the systematics of baryon stopping to understand the mechanisms of energy deposition. Ultimately, one would like to understand all of this physics in relation to the microscopic processes suggested by QCD. In the meantime, the elegant structure of the data itself may well point theory in completely new directions or suggest unexpected connections between various techniques.

\section{Acknowledgements}

The author would like to thank the organizers of the LHC07 workshop, Jan Rak and Kari Eskola, for the invitation to speak (twice!). Special thanks to Vesa Ruuskanen for stimulating discussions in Jyväskylä, for reviewing this manuscript and for bringing up the potential conflict of longitudinal size and thermal wavelength at the LHC. 


\section{References}

[1] P. F. Kolb and U. W. Heinz, arXiv:nucl-th/0305084.

[2] J. D. Bjorken, Phys. Rev. D 27, 140 (1983).

[3] E. Fermi, Prog. Theor. Phys. 5, 570 (1950).

[4] L. D. Landau, Izv. Akad. Nauk Ser. Fiz. 17, 51 (1953).

[5] S. Z. Belenkij and L. D. Landau, Nuovo Cim. Suppl. 3S10, 15 (1956) [Usp. Fiz. Nauk 56, 309 (1955)].

[6] P. Carruthers, Annals N.Y.Acad.Sci. 229, 91 (1974).

[7] T. Hirano and M. Gyulassy, Nucl. Phys. A 769, 71 (2006).

[8] T. Sjostrand, arXiv:hep-ph/9508391.

[9] A. H. Mueller, Nucl. Phys. B 228, 351 (1983).

[10] A. Moraes, C. Buttar and I. Dawson. SN-ATL-2006-057, CERN Document (ATLAS Scientific Note Public) August 2006. To be published in The European Physics Journal Direct.

[11] T. Sjostrand, S. Mrenna and P. Skands, JHEP 0605, 026 (2006).

[12] G. Marchesini, B. R. Webber, G. Abbiendi, I. G. Knowles, M. H. Seymour and L. Stanco, Comput. Phys. Commun. 67, 465 (1992).

[13] R. Engel, Z. Phys. C 66, 203 (1995).

[14] M. Gyulassy and X. N. Wang, Comput. Phys. Commun. 83, 307 (1994).

[15] W. Busza and A. S. Goldhaber, Phys. Lett. B 139, 235 (1984).

[16] M. Basile et al., Phys. Lett. B 92, 367 (1980).

[17] A. E. Brenner et al., Phys. Rev. D 26, 1497 (1982).

[18] M. Batista and R. J. M. Covolan, Phys. Rev. D 59, 054006 (1999).

[19] F. Cooper, G. Frye and E. Schonberg, Phys. Rev. Lett. 32, 862 (1974).

[20] B. B. Back et al., Phys. Rev. Lett. 91, 052303 (2003).

[21] P. Carruthers and M. Doung-van, Phys. Rev. D 8, 859 (1973).

[22] P. Steinberg, Acta Phys. Hung. A 24, 51 (2005).

[23] B. B. Back et al., Nucl. Phys. A 757, 28 (2005).

[24] K. Tesima, Z. Phys. C 47, 43 (1990).

[25] W. Busza, Acta Phys. Polon. B 35, 2873 (2004).

[26] D. V. Minh and P. Carruthers,Phys. Rev. Lett. 31, 133 (1973).

[27] S. S. Adler et al. , Phys. Rev. Lett. 91, 241803 (2003).

[28] J. Adams et al. , Phys. Rev. Lett. 91, 172302 (2003).

[29] Z. W. Lin, C. M. Ko, B. A. Li, B. Zhang and S. Pal, Phys. Rev. C 72, 064901 (2005).

[30] I. G. Bearden et al., Phys. Rev. Lett. 94, 162301 (2005). 
[31] B. B. Back et al., Phys. Rev. C 74, 021901 (2006).

[32] P. Steinberg, arXiv:nucl-ex/0703002.

[33] B. B. Back et al., Phys. Rev. C 74, 021902 (2006).

[34] I. G. Bearden et al., Phys. Rev. Lett. 93, 102301 (2004).

[35] J. Cleymans, M. Stankiewicz, P. Steinberg and S. Wheaton, arXiv:nucl-th/0506027.

[36] P. Steinberg, PoS(CPOD2006)036, arXiv:nucl-ex/0702019.

[37] P. Steinberg, Nucl. Phys. A 752, 423 (2005).

[38] M. Gyulassy and L. McLerran, Nucl. Phys. A 750, 30 (2005).

[39] P. Kovtun, D. T. Son and A. O. Starinets, Phys. Rev. Lett. 94, 111601 (2005).

[40] D. Teaney, Phys. Rev. C 68, 034913 (2003). 